\title{
Discriminating a deep gallium antisite defect from shallow acceptors in GaAs using supercell calculations
}

\author{
Peter A. Schultz* \\ Multiscale Science, Sandia National Laboratories, Albuquerque, New Mexico 87185-1322, USA \\ (Received 22 September 2015; revised manuscript received 4 February 2016; published 1 March 2016)
}

\begin{abstract}
For the purposes of making reliable first-principles predictions of defect energies in semiconductors, it is crucial to distinguish between effective-mass-like defects, which cannot be treated accurately with existing supercell methods, and deep defects, for which density functional theory calculations can yield reliable predictions of defect energy levels. The gallium antisite defect $\mathrm{Ga}_{A s}$ is often associated with the 78/203 meV shallow double acceptor in Ga-rich gallium arsenide. Within a conceptual framework of level patterns, analyses of structure and spin stabilization can be used within a supercell approach to distinguish localized deep defect states from shallow acceptors such as $\mathrm{B}_{A s}$. This systematic approach determines that the gallium antisite supercell results has signatures inconsistent with an effective mass state and cannot be the 78/203 shallow double acceptor. The properties of the $\mathrm{Ga}$ antisite in GaAs are described, total energy calculations that explicitly map onto asymptotic discrete localized bulk states predict that the Ga antisite is a deep double acceptor and has at least one deep donor state.
\end{abstract}

DOI: 10.1103/PhysRevB.93.125201

\section{INTRODUCTION}

A comprehensive and detailed understanding of the possible defects and their chemistry-formation energies, diffusion activation energies, defect energy levels-is necessary for quantitatively predictive modeling of processing and radiation effects in semiconductors such as GaAs [1,2]. First-principles electronic structure simulations are crucial to elucidating this defect physics, because, while some of the requisite atomic scale physics is available through experimental measurement $[3,4]$, much is experimentally inaccessible or unknown, and can only be discovered and characterized through firstprinciples calculations [5].

Substantial mysteries in the defect physics of GaAs remain despite decades of study. Computations proved pivotal in identifying the EL2 midgap defect center in GaAs as the arsenic antisite [6,7], but definitive identification of other intrinsic defects in GaAs has lagged because of both insufficiently discriminating experiment $[3,4]$ and insufficiently predictive computational methods. The arsenic antisite $\mathrm{As}_{G a}$, an arsenic replacing a gallium atom in the zinc-blende lattice, might be well characterized, but the gallium antisite $\mathrm{Ga}_{A s}$ is not.

Experiments identified a shallow double acceptor with levels at 78 and $203 \mathrm{meV}$ above the band edge in Ga-rich GaAs [8-10] and made the natural inference this was the gallium antisite that would presumably be more prevalent in Ga-rich grown material. Recent experiments contradict this assignment [11], instead implicating the boron antisite $\mathrm{B}_{A s}$ (boron replacing an arsenic in the lattice) as responsible for this defect.

A computational investigation of defects in GaAs by Schultz and von Lilienfeld [5] alluded to this uncertainty

\footnotetext{
*paschul@sandia.gov

Published by the American Physical Society under the terms of the Creative Commons Attribution 3.0 License. Further distribution of this work must maintain attribution to the author(s) and the published article's title, journal citation, and DOI.
}

surrounding $\mathrm{Ga}_{A s}$. They judged $\mathrm{Ga}_{A s}$ to be a deep defect based upon computed changes in bond distances around the antisite as a function of charge states from $(2-)$ to $(2+)$. Komsa and Pasquarello, using hybrid functionals, echoed this result, finding deep states from midgap to the valence band edge (VBE) [12]. However, an alternative interpretation based on a bounds analysis from Wright and Modine [13] recently asserted that $\mathrm{Ga}_{A s}$ is instead a shallow double acceptor, supporting the original experimental hypothesis that the 78/203 defect was the gallium antisite.

Accurate prediction of shallow, effective-mass-like defect levels is still impossible with supercell methods and stateof-the-art density functional theory (DFT) methods [14]. The strong interaction between periodic images in the defect cause strong banding in the defect state, and it proves difficult to distinguish defect states from bulk band states in smaller supercells. Huge computational supercell models would be necessary to encompass the Rydberg-like hydrogenic state, well beyond the 1000-atom supercell models tractable in conventional DFT codes. It is unclear whether existing DFT can quantify these well even if supercell calculations were extended to such extreme computational models [14].

On the other hand, for localized defect states, well contained within the volume of a supercell, accuracy of $0.1-0.2 \mathrm{eV}$ in defect levels over a wide survey of different defects is achievable for semiconductors such as Si [15] and GaAs [5,16] with DFT supercell calculations. Hence, for the purposes of making reliable predictions with first-principles methodsand assessing how reliable those predictions are-it becomes crucial to distinguish between localized defects, for which accurate quantitative results can be obtained, and effectivemass-like states, for which supercell calculations fail.

In this paper I systematically probe this distinction for the $\mathrm{Ga}_{A s}$ defect in GaAs within a supercell approximation and DFT, to determine the conditions that demonstrate that it is deep defect - or not - and quantitatively characterize the $\mathrm{Ga}_{A s}$ defect. There is significant banding of the defect states even in the largest 1000-atom supercells, with making unambiguous characterization of these states as deep, shallow, or below 


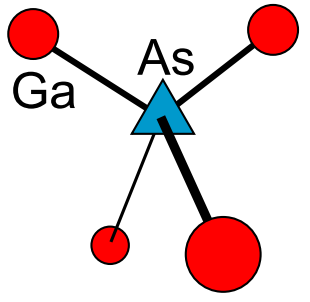

(a)

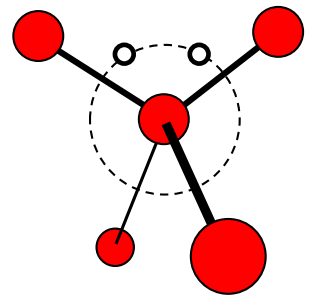

(b)
FIG. 1. Schematic illustration of the Ga antisite. The As in the perfect crystal (a) is replaced by a Ga in the lattice (b), leaving behind two holes in the bonds, having two fewer valence electrons than the As it replaces.

the band edge unclear. Within a level occupation analysis that asymptotically maps onto the infinitely dilute limit, it is shown that the changes as the supercell increases can be used to distinguish shallow from deep defect character, where the results of any single supercell size would be ambiguous.

The paper begins with describing the background surrounding the $\mathrm{Ga}_{A s}$ and the 78/203 shallow double acceptor in GaAs. It continues with describing the characteristics that are exhibited within a supercell approximation for shallow and deep defects, factoring in finite size effects, and how these might be assessed in a practical computational supercell study. The $\mathrm{Ga}_{A s}$ is determined to be unambiguously distinct from an effectivemass-like defect such as $\mathrm{B}_{A s}$. The computational analysis indicates that the $\mathrm{Ga}_{A s}$ is a deep double acceptor, and additionally possesses one deep donor state and likely a second, perhaps shallow donor state. $\mathrm{Ga}_{A s}$ cannot be the 78/203 meV double acceptor seen in Ga-rich GaAs, confirming the most recent experimental findings from Kiessling et al. [11].

\section{BACKGROUND}

The EL2 is the dominant defect in typical GaAs, leading to semi-insulating behavior in as-grown undoped material, the EL2 donor state pinning the Fermi level near midgap. Grown from a very Ga-rich melt, greater than $\sim 0.53 \mathrm{Ga}$ mole fraction, the resistivity is instead $p$ type, accompanied by the appearance of a shallow double acceptor, with levels at 78 and $203 \mathrm{meV}$ above the VBE [8-10]. This shallow double acceptor grows into the native undoped GaAs using liquid encapsulated Czochralski (LEC), liquid phase epitaxy (LPE), or vertical gradient freeze (VGF) technologies [8,11].

From the lack of level splittings in the observed spectra, Elliott et al. [8,10] determined that this shallow acceptor must possess tetrahedral symmetry [8]. Appearing in Ga-rich material that is not (intentionally) doped, this residual defect was deemed to likely be intrinsic. As illustrated in Fig. 1, the neutral gallium antisite, with two fewer electrons than the arsenic atom it replaces, introduces two holes into the bond network. This can capture two electrons, and $\mathrm{Ga}_{A s}$ is expected to be a double acceptor. The involvement of boron in the defect, isovalent with gallium, would also create a double acceptor, and could not be totally excluded, but Elliott et al. [8,10] noted a lack of correlation between boron content and the acceptor concentration. From these observations - as grown Ga-rich, likely intrinsic, tetrahedral symmetry, double acceptor-the natural inference was the shallow double acceptor was associated with the $\mathrm{Ga}$ antisite.

Apprehensions about the potential role of boron in the defect expressed in the early work $[8,10]$ lingered. The defect was either the $\mathrm{B}$ antisite, or the $\mathrm{Ga}$ antisite $[17,18]$. The data indicated only one of these could be present as a shallow double acceptor, otherwise, where was the second set of acceptor levels that would indicate the coexistence of both [18]? The infrared experiments of Addinall and co-workers [19,20] cast doubt on the $\mathrm{Ga}_{A s}$ assignment, correlating the $78 / 203$ defect with $\mathrm{B}_{A s}$ from infrared experiments. This association of the 78/203 with $\mathrm{B}_{A s}$ was also supported by Suezawa and Sumino [21].

Boron is unavoidably present in standardly grown GaAs, incorporating from the crucible and the $\mathrm{B}_{2} \mathrm{O}_{3}$ cap that protects the melt [11]. Kiessling and co-workers [11] used a vapor-pressure-controlled Czochralski method to grow GaAs without the $\mathrm{B}_{2} \mathrm{O}_{3}$ encapsulant, removing the source of boron contamination in the undoped GaAs. Their resulting material was semi-insulating, just as in As-rich material, rather than $p$ type, as in the conventionally grown Ga-rich material. The residual double acceptor was absent, apparently implicating boron in the formation of the 78/203 defect.

The crux of this extended experimental debate is lack of unambiguous evidence that eliminates boron as a player in this shallow defect, compounded by a lack of direct observation and knowledge about the gallium antisite. Computational approaches have been similarly inconclusive, mostly neglecting the $\mathrm{Ga}$ antisite in GaAs.

In their comprehensive study of simple intrinsic defects in GaAs [5], Schultz and von Lilienfeld included the $\mathrm{Ga}$ antisite, probing $\mathrm{Ga}_{A s}$ charge states ranging from the (2-) double acceptor to a $(2+)$ double donor state, focused mostly on $T_{d}$ structures. For the $\mathrm{Ga}$ interstitial $\mathrm{Ga}_{i}$, localized charge transitions triggered significant changes in bond distances, while charge transitions ascribed to delocalized states led to negligible changes in bond distances around $\mathrm{Ga}_{i}$. For $\mathrm{Ga}_{A s}$, the nearest neighbor $\mathrm{Ga}-\mathrm{Ga}$ distance changed significantly with every transition from (2-) to $(2+)$, becoming progressively longer as electrons were removed. An effective-mass-like state would not occasion significant local structural changes, indicating these were localized deep defect states. While noting that small symmetry-lowering distortions were favored, this study did not explore the distorted potential energy surfaces for $\operatorname{Ga}_{A s}(q)$.

A hybrid functional study on vacancies and antisites in GaAs [12], using smaller $2 \times 2 \times 2$ 64-atom cubic cells and $2^{3} k$ sampling, also investigated $\mathrm{Ga}_{A s}$. The hybrid exchange mixing was tuned to the experimental band gap, ostensibly to eliminate errors associated with gap underestimation. Their computed defect levels mimicked the earlier results using semilocal functionals: a sequence of deep energy levels beginning with a midgap $(2-/ 1-)$ charge transition culminating with a $(1+/ 2+)$ level near the VBE. Additionally, Schultz and Edwards [22] found that the $\mathrm{Ga}_{A s}$ defect levels computed from total energy differences changed very little using different semilocal functionals, or alternate pseudopotentials that included the Ga $3 d$ electrons in the valence, despite DFT band gaps as $0.1 \mathrm{eV}$ or as large as $0.8 \mathrm{eV}$. Computed $\mathrm{Ga}_{A s}$ levels obtained from total energy differences are insensitive to the DFT band gap. 


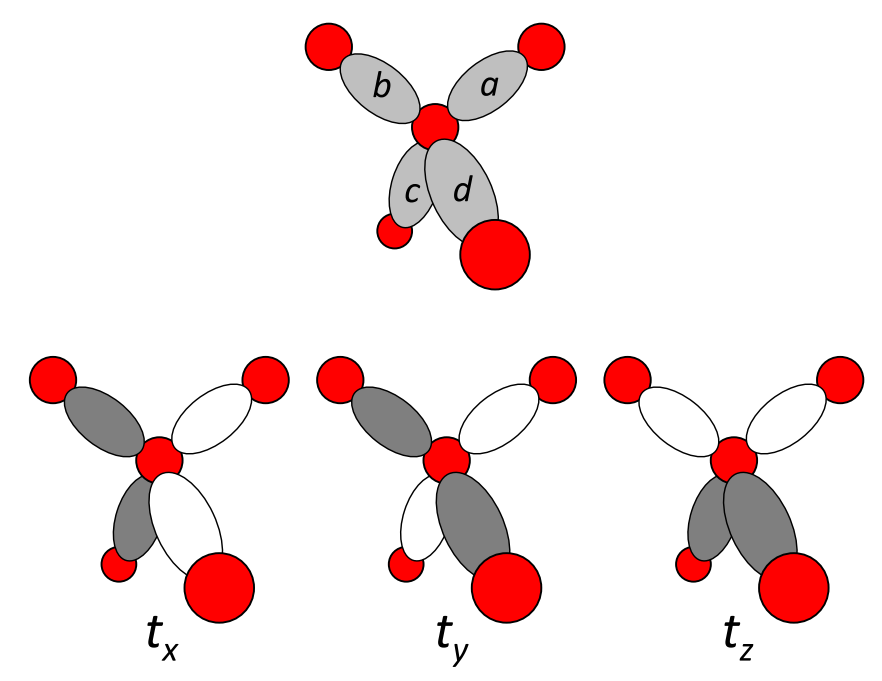

FIG. 2. Schematic illustration of electronic structure around $\mathrm{Ga}_{A s}$ in GaAs, within a LCAO-MO picture. Denoting the bonds as $a, b$, $c$, and $d$, the holes will present in the degenerate tripleton of $t_{2}$ orbitals, which can be represented as $p$-like orbitals, as $t_{x}, t_{y}$, and $t_{z}$. The symmetric $a_{1}$ combination, lacking a node, will be more strongly bound and not be involved in the defect levels. Gray and white shading in the orbitals denote the relative phases in the orbitals.

A recent study using the local density approximation (LDA) and large-core pseudopotentials, however, came to a contrary conclusion [13]. Positing $\mathrm{Ga}_{A s}$ kept a $T_{d}$ structure, it computed nominal $\mathrm{Ga}_{A s}$ defect levels in 216-, 512-, and 1000-atom cubic supercells. Interpreting these results within a bounds-analysis approach, Wright and Modine [13] asserted the two $\mathrm{Ga}_{A s}$ acceptor states were shallow hydrogenic states, and that the donor states were delocalized band states rather than true defect states. This interpretation was consistent with inferences made from earlier experiments [8-10] and disagreed with the other computational results $[5,12]$.

To determine whether the acceptor states for $\mathrm{Ga}_{A s}$ are deep/midgap or shallow/effective mass requires that this assessment of the state character in DFT calculations be made with greater confidence. A principal goal of this paper is to examine more carefully how to discriminate whether $\mathrm{Ga}_{A s}$ is a shallow state or a deep state, given that finite size effects inherent to the supercell approximation can make it challenging to make this distinction within computational DFT.

\section{THEORY}

Following the example of Watkins' insightful analyses of defects in silicon [23], the electronic structure of the Ga antisite is conveniently and usefully described within a molecular orbital (MO) model based on a linear combination of atomic orbitals description (LCAO). Adopting the bond orbitals to its neighbors as the "atomic orbitals," denoted as $a, b, c$, and $d$ in Fig. 2, the bond orbitals surrounding $\mathrm{Ga}_{A s}$ can be recast into four (unnormalized) symmetry-adapted molecular orbitals (MO) within $T_{d}$ symmetry: an $s$-like $a_{1} \mathrm{MO}, a+b+c+d$, and three $p$-like $t_{2}$ MOs, $t_{z}=(a+b)-(c+d), t_{y}=(a+$ $c)-(b+d), t_{x}=(a+d)-(b+c)$, as depicted in Fig. 2.

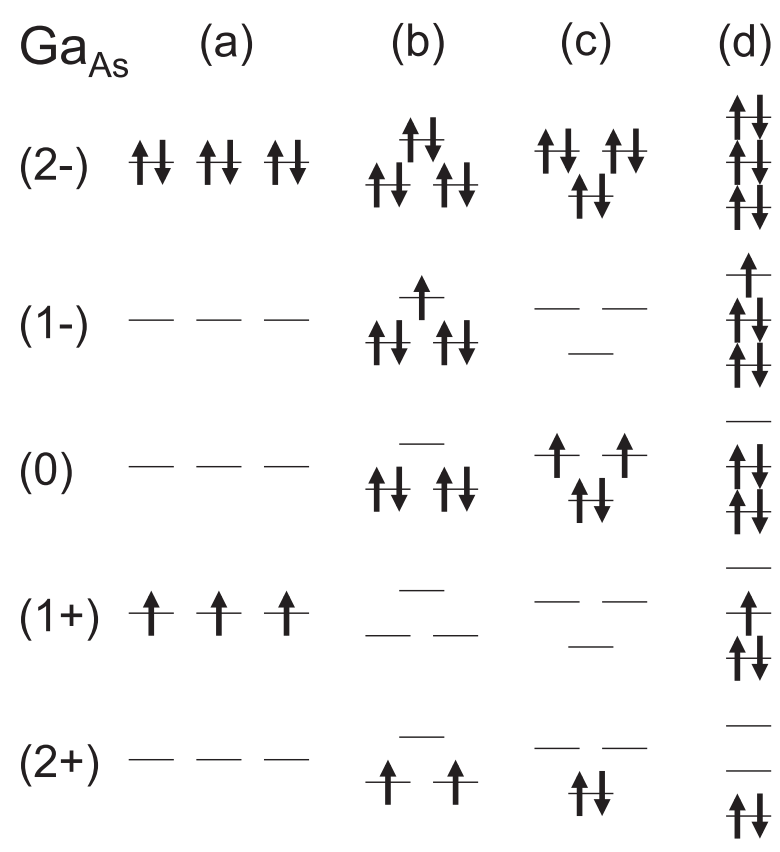

FIG. 3. Level and occupation patterns for proper (nondegenerate) total states of $\mathrm{Ga}_{A s}$ in GaAs. In $\mathrm{Ga}_{A s}(2-)$, (a) the tripleton of degenerate $t_{2}$ orbitals are full, and the total state is nondegenerate. Symmetry lowerings from $T_{d}$ can cause (b) a singleton orbital to rise above a doubleton, as in the resonant $D_{2 d}$ (or $C_{3 v}$ ) or (c) to be drawn below a doubleton, as in paired $D_{2 d}$, or (d) to create three nondegenerate singletons, as with a $C_{2 v}$ distortion. The charge state sets the number of electrons that fill these level patterns, describing the allowed structural spin states that define a proper nondegenerate total state.

The substitution of Ga for As perturbs the local potential, the weaker potential of the Ga causing the electrons in these bonds to be less bound than in the perfect crystal. The $s$-like combination maps onto states embedded below the VBE. The three $p$-like states map onto states at the VBE for the perfect crystal, causing the Ga to be an acceptor. For the double acceptor, the $\mathrm{Ga}_{A s}(2-)$, all of these states are full, and the Ga antisite retains the full $T_{d}$ symmetry of the perfect lattice.

In $\mathrm{Ga}_{A s}(1-)$, one electron is missing from the $t_{2}$ states, and $\mathrm{Ga}_{A s}(0)$ is missing two electrons. The first electron comes from one of three degenerate $t_{2}$ orbitals, creating a degenerate total electronic state. This electronic degeneracy couples to an orientation change, using a symmetry-reducing distortion to split the degeneracy, driving occupied orbitals deeper and the open shell orbitals higher. This is commonly termed a Jahn-Teller (JT) effect [24].

Illustrated in Fig. 3, only a small number of symmetryreduced level patterns are possible starting with this (a) degenerate tripleton of $t_{2}$ orbitals, (b) splitting one orbital above a doubleton of degenerate orbitals, (c) splitting a doubleton above a singleton, or (d) splitting into three nondegenerate orbital eigenstates.

Figure 4 illustrates possible distortions around the $\mathrm{Ga}_{A s}$. A tetragonal distortion compressing bonds along the $z$ axis, a resonant-bond $D_{2 d}\left(\mathrm{r} D_{2 d}\right)$ structure, splits one orbital upwards, intensifying the node in the $t_{z}$ state, destabilizing it with respect to the other $t_{2}$ states. The other tetragonal distortion, a 


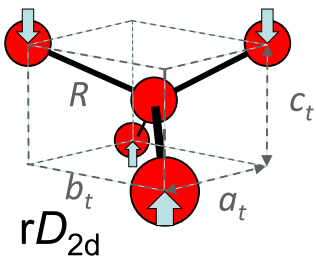

(a)

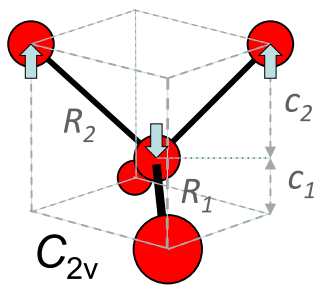

(c)

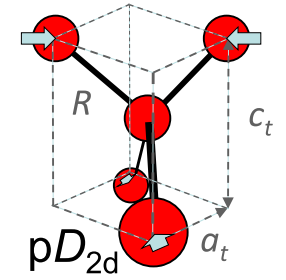

(b)

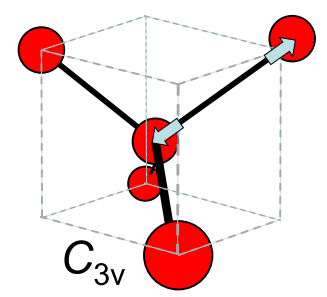

(d)
FIG. 4. Schematic depictions of primary structural distortions of the Ga antisite in GaAs: (a) A resonant-bond $D_{2 d}$, where a tetragonal distortion compresses along the $z$ axis to make $c_{t}$ shorter than than $a_{t}\left(=b_{t}\right)$; (b) a pairied $D_{2 d}$, where the tetragonal distortion makes $a_{t}$ $\left(=b_{t}\right)$ shorter than $c_{t}$; (c) a $C_{2 v}$ distortion, where the antisite moves toward one side, the bonds to one side becoming shorter than the other; and (d) a $C_{3 v}$ distortion where one bond becomes longer than the other three.

pairing $D_{2 d}\left(\mathrm{p} D_{2 d}\right)$, stabilizes one orbital below a doubleton. These doubleton-singleton patterns also result from trigonal $C_{3 v}$ distortions. A $C_{2 v}$ distortion, where the antisite is shifted off-origin along a Cartesian axis, results in three nondegenerate orbitals, as will a distortion of any lower symmetry.

This analysis determines which level patterns are consistent with a nondegenerate state for a given number of electrons, a guide to which distortions are plausible candidates in each charge state. The level and occupancy pattern indicates the spin states appropriate to construct a proper total state.

In Fig. 3 the implications for different Ga antisite states are illustrated. For the $\mathrm{Ga}_{A s}(2-)$, all the $t_{2}$ orbitals are full, there is no overall state degeneracy, and the $(2-)$ defect can retain its $T_{d}$ symmetry.

For the $\mathrm{Ga}_{A s}(1-)$, one orbital is half-filled. To create a nondegenerate state, the highest level must be a singleton (the lower two states are full, and could either be a degenerate doubleton or two singletons). This is compatible with a $\mathrm{r} D_{2 d}$, as in Fig. 4(a), or a $C_{3 v}$ distortion, or a $C_{2 v}$ or lower symmetry.

For $\mathrm{Ga}_{A s}(0)$, removing a second electron from the same orbital also creates a nondegenerate state, and should accentuate any distortion. Another possibility is using the level pattern of Fig. 3(c), consistent with the $\mathrm{p} D_{2 d}$ distortion, removing one electron from each orbital in the higher doubleton. This must be a spin triplet (the proper singlet spin state of two open shell orbitals is not accessible within standard DFT) to avoid a JT instability.

$\mathrm{A} \mathrm{Ga}_{A s}(1+)$ state with a singleton-doubleton level pattern (either $D_{2 d}$ or $C_{3 v}$ ) puts either one electron or one hole in double-degenerate orbitals, an improper state subject to JT instability. A proper nondegenerate state is possible populating three nondegenerate orbitals with three electrons, where the middle orbital level is half-occupied (e.g., a $C_{2 v}$ structure). A second proper occupation level pattern is possible in the $T_{d}$ structure, occupying each of the three degenerate $t_{2}$ states by a single electron, in a high-spin $\left(\frac{3}{2}\right)$ state.

The $\mathrm{Ga}_{A s}(2+)$ places two electrons among the three defect orbitals, viable if the level pattern has a nondegenerate orbital level below two empty orbitals (either $C_{2 v}$ or $\mathrm{p} D_{2 d}$ ), or with two triplet coupled electrons in doubleton orbitals, as with a $\mathrm{r} D_{2 d}$ structure.

While an averaged description in the $T_{d}$ symmetry is often used to describe all these charge states, except for (2-), these are not proper discrete defect states. The level occupation patterns in Fig. 3 describe the defect states available within DFT (we ignore spin-orbit effects in this discussion, as DFT calculations typically used for GaAs defects, like here, ignore these). A purposeful assessment of defect stability with charge state must explore these patterns. While spin effects are shown to be not quantitatively important in GaAs defect (generally $50 \mathrm{meV}$ or less) [5], they are crucial in qualitatively discriminating the nature of the various $\mathrm{Ga}_{A s}$ charge states. A JT motivated occupation level pattern provides a guide to characterize and screen the different defect states.

The presence of a JT degeneracy does not predict the sense of the mandated distortion, nor does it speak to the magnitude of an optimal distortion. For a deep defect whose electronic state is strongly localized around the defect, the distortion will generally be larger; for a shallow defect with a diffuse electronic state, the driving force for a distortion will be less, and the distortion smaller. The removal/addition of electrons to these states will engender greater (lesser) changes in the local structure for a more (less) localized state.

Finite cell effects obscure this analysis for shallow defects. Viability of a supercell approach [25] is predicated on the assumption the defect state is well contained within the volume of the supercell. The ideal isolated defect has a discrete defect level, without any dispersion. In a finite supercell, the interaction with its periodic defect neighbors causes defect banding. A strongly localized state will have minimal interactions with its artificial periodic images. A delocalized state magnifies the spurious dispersion in the defect states. For a delocalized state that is poorly contained within the volume of a supercell, especially in the limit of a shallow donor or shallow acceptor, the computed state might be dominated by the artificial interaction between the periodic defect states, to the point where it might be difficult to distinguish the shallow state from a band state. Earlier work has shown [14] that supercells of at least 64000 atoms are necessary to resolve shallow states from bulk, well beyond the capabilities of current DFT codes.

\section{COMPUTATIONAL METHODS}

The DFT calculations in this study are executed using SEQQUEST [26], a pseudopotential code for performing largescale supercell calculations with a local orbital basis. The methods are generally the same as those used in our earlier study of simple intrinsic defects in GaAs [5]. The defect calculations here use both the LDA [27] and the PerdewBurke-Ernzerhof (PBE) flavor of the generalized gradient 
approximation [28]. The different behaviors of these two functionals can be illuminating concerning the nature of a defect [29]. While the spin-polarization energy for GaAs defects is quantitatively modest and can usually be neglected within the physical uncertainties of DFT functionals [5], for the purposes elucidating the nature of $\mathrm{Ga}_{A s}$ spin (and the difference between LDA and PBE) proves crucial and is included here. The results are mostly insensitive to whether the $3 d$ electrons are included in the valence electrons or placed in the core of the pseudopotential (PP), hence, the large-core (3d in core) PP $[5,30]$ with nonlinear core corrections [31] are used in this work to enable calculations of the largest supercell models efficiently. Well-converged double- $\zeta$ plus polarization, contracted-Gaussian basis sets are used on all atoms.

The computational models are $3 \times 3 \times 3,4 \times 4 \times 4$, and $5 \times 5 \times 5$ scaled versions of the conventional 8 -atom cubic cell of the zinc-blende lattice, supercells containing 216, 512, and 1000 atoms in the perfect crystal, respectively. The computed bulk lattice parameter for LDA and PBE, 5.628 and $5.739 \AA$, respectively, are used in all calculations. A $2^{3} k$-point sample is used for all these supercells, a sampling demonstrated to be converged in the 216-atom supercell $\left(2^{3} k\right.$ sampling leads to even denser, more converged sampling for larger supercells). Atomic positions are relaxed until forces are less than 0.0002 Ry/bohr $(0.005 \mathrm{eV} / \AA)$ on every atom in a relaxed ground state structure, good enough to resolve total energies to within a couple of meV. A discrete defect occupation [15] is applied for every defect, to help ensure that state occupations are consistent with the asymptotic dilute defect occupations.

Rigorous boundary conditions for all the quoted results from charged defect supercell calculations are enforced with the local moment countercharge (LMCC) method [32,33], selfconsistently incorporating the correct asymptotic electrostatic potential due to an isolated charge. Defect energies and levels are computed from total energy differences, rigorously avoiding spurious charge-charge interactions across supercell boundaries, referencing the charge within the supercell to a common electron reservoir, and explicitly incorporating the polarization energy of long range screening response to the charge [15].

The numerical results were validated to reproduce the measured detailed defect levels in Si to within $\sim 0.1 \mathrm{eV}$ across the band gap [15]. In GaAs, the spread of computed defect levels [22] matches the experimental gap [34]. The two $\mathrm{As}_{G a}$ donor levels were computed to lie midgap and $\sim 0.25 \mathrm{eV}$ below, inside the uncertainty defined by the scatter of different experimental measurements for EL2. Based on computed defect level energies, these results were able to reassign the observed $E 1-E 2$ and the $E 3$ defect centers to the divacancy and a bistable arsenic vacancy, respectively [16], the predicted levels again agreeing with experimental measurements within $0.1 \mathrm{eV}$. This lends confidence to the quantitative analysis of defect energies in GaAs and the current results for $\mathrm{Ga}_{A s}$.

\section{RESULTS AND ANALYSIS}

\section{A. Tetrahedral structures}

A preliminary screen for the $\mathrm{Ga}_{A s}$ constrains the structure to a $T_{d}$ symmetry, an assumption whose quantitative validity
TABLE I. Computed Ga-Ga bond distance, in $\AA$, for $T_{d} \mathrm{Ga}_{A s}$ with supercell and functional.

\begin{tabular}{|c|c|c|c|c|}
\hline & \multirow{2}{*}{$\frac{\text { Spinless }}{3 \times 3 \times 3^{\mathrm{a}}}$} & \multicolumn{3}{|c|}{ High spin } \\
\hline & & $3 \times 3 \times 3$ & $4 \times 4 \times 4$ & $5 \times 5 \times 5$ \\
\hline \multicolumn{5}{|l|}{ LDA } \\
\hline $\mathrm{Ga}_{A s}(2-)$ & 2.329 & 2.329 & 2.329 & 2.330 \\
\hline $\operatorname{Ga}_{A s}(1-)$ & 2.364 & 2.365 & 2.366 & 2.366 \\
\hline $\mathrm{Ga}_{A s}(0)$ & 2.400 & 2.397 & 2.397 & 2.397 \\
\hline $\mathrm{Ga}_{A s}(1+)$ & 2.416 & 2.449 & 2.436 & 2.432 \\
\hline $\mathrm{Ga}_{A s}(2+)$ & 2.436 & 2.461 & 2.440 & 2.430 \\
\hline \multicolumn{5}{|l|}{ PBE } \\
\hline $\mathrm{Ga}_{A s}(2-)$ & 2.389 & 2.389 & 2.394 & 2.394 \\
\hline $\mathrm{Ga}_{A s}(1-)$ & 2.431 & 2.433 & 2.434 & 2.435 \\
\hline $\mathrm{Ga}_{A s}(0)$ & 2.467 & 2.480 & 2.479 & 2.480 \\
\hline $\mathrm{Ga}_{A s}(1+)$ & 2.498 & 2.551 & 2.539 & 2.537 \\
\hline $\mathrm{Ga}_{A s}(2+)$ & 2.528 & 2.564 & 2.543 & 2.536 \\
\hline
\end{tabular}

${ }^{\mathrm{a}}$ From Ref. [5].

depends upon weak JT distortions. Without spin, sequential removal of electrons from the $(2-)$ charge state through the $(2+)$ charge state causes the $\mathrm{Ga}-\mathrm{Ga}$ bond distance to increase, implying a localized removal of charge indicative of a localized defect transition for each charge change [5].

Table I augments this analysis with spin-polarized DFT. The high-spin state for each charge state is used, dictated by the number of open shells when populating a tripleton of degenerate $t_{2}$ orbitals: the $(1-)$ state has a single open shell, and one unpaired electron, the $(0)$ has two unpaired electrons in a spin triplet, and the $(1+)$, three unpaired electrons. With four missing electrons in $\operatorname{Ga}_{A s}(1+)$, only two electrons are distributed in three $t_{2}$ orbitals, and a spin triplet is the high-spin state.

Spin polarization alters the bond-length-motivated assessment. Without spin polarization, the $\mathrm{Ga}-\mathrm{Ga}$ bond distance incrementally increases from (2-) through $(2+)$. With spin polarization, these increases stop abruptly stop at $(1+)$, using both LDA and PBE. This is most apparent in the largest 1000atom supercell. The spin-resolved bond distance analysis only shows evidence for localized states through $(1+)$, rather than $(2+)$ indicated with functionals without spin polarization [5].

\section{B. Distorted structures}

The $\mathrm{Ga}_{A s}(2-)$ has no cause to distort from the symmetric $T_{d}$ structure, all antisite bonds are full. The search for loweredsymmetry distortions in other charge states found structures illustrated in Fig. 4: tetragonally distorted $\mathrm{r} D_{2 d}$ and $\mathrm{p} D_{2 d}$ structures, a $C_{2 v}$ structure where the Ga antisite shifts along a Cartesian ( $z$ ) axis, and trigonally distorted $C_{3 v}$.

The $\mathrm{Ga}_{A s}(1-)$ is missing one electron from its bonds, leading to a triply degenerate total state. A spinless relaxation finds the tetragonally distorted $\mathrm{r} D_{2 d}$ to be the lowest energy structure for both LDA and PBE, with an energy-lowering from the symmetric $T_{d}$ of $\sim 10-30 \mathrm{meV}$ (depending on supercell size and functional, the "simulation context"), while the $C_{2 v}$ is 5-10 meV higher than the $\mathrm{r} D_{2 d}$. The $\mathrm{p} D_{2 d}$, and $C_{3 v}$ distortions also lead to energy lowering, demonstrating the instability in the JT degeneracy in the $T_{d}$, but are JT degenerate states themselves (one missing electron in a degenerate doubleton). 
TABLE II. Computed structural distortions (unitless) and bond distances $(\AA)$ for $\mathrm{Ga}_{A s}$, spin polarized where eligible. See Fig. 4 for description of the distortion parameters.

\begin{tabular}{|c|c|c|c|c|c|c|c|c|c|c|c|}
\hline \multirow[b]{2}{*}{ Supercell } & \multicolumn{2}{|c|}{$\mathrm{r} D_{2 d}(1-)$} & \multicolumn{2}{|c|}{$\mathrm{r} D_{2 d}(0)$} & \multicolumn{2}{|c|}{$\mathrm{p} D_{2 d}(0)$} & \multicolumn{3}{|c|}{$C_{2 v}(0)$} & \multicolumn{2}{|c|}{$\mathrm{r} D_{2 d}(2+)$} \\
\hline & $c_{t} / a_{t}$ & $R$ & $c_{t} / a_{t}$ & $R$ & $c_{t} / a_{t}$ & $R$ & $c_{2} / c_{1}$ & $R_{1}$ & $R_{2}$ & $c_{t} / a_{t}$ & $R$ \\
\hline \multicolumn{12}{|l|}{ LDA } \\
\hline $3 \times 3 \times 3$ & 0.949 & 2.423 & 0.898 & 2.402 & 1.006 & 2.401 & 1.243 & 2.345 & 2.462 & 0.954 & 2.457 \\
\hline $4 \times 4 \times 4$ & 0.935 & 2.416 & 0.892 & 2.402 & 1.039 & 2.400 & 1.299 & 2.339 & 2.480 & 0.965 & 2.441 \\
\hline $5 \times 5 \times 5$ & 0.930 & 2.415 & 0.886 & 2.404 & 1.059 & 2.404 & 1.322 & 2.338 & 2.480 & 0.966 & 2.433 \\
\hline \multicolumn{12}{|l|}{ PBE } \\
\hline $3 \times 3 \times 3$ & 0.936 & 2.508 & 0.894 & 2.476 & 1.010 & 2.482 & 1.274 & 2.408 & 2.559 & 0.973 & 2.568 \\
\hline $4 \times 4 \times 4$ & 0.927 & 2.502 & 0.881 & 2.478 & 1.066 & 2.487 & 1.350 & 2.403 & 2.586 & 0.980 & 2.547 \\
\hline $5 \times 5 \times 5$ & 0.923 & 2.501 & 0.876 & 2.480 & 1.075 & 2.489 & 1.361 & 2.402 & 2.590 & 0.986 & 2.537 \\
\hline
\end{tabular}

These would distort further (differently) to remove this degeneracy. In the $(1-)$ charge state, the $C_{2 v}$ and $\mathrm{p} D_{2 d}$ are lower in energy and the likely candidates.

With an odd electron, $\mathrm{Ga}_{A s}(1-)$ should be described with spin polarization. For the $\mathrm{r} D_{2 d}$ ground state, the LDA spin stabilization is modest, 19-24 meV, while the PBE spin stabilization is larger, $33-41 \mathrm{meV}$, depending on simulation context. Including these spin-polarization energies results in a total symmetry lowering from $T_{d}$ of $62 \mathrm{meV}$ in the LDA and $71 \mathrm{meV}$ with the PBE, for the largest supercells.

This symmetry-lowering increases with supercell size, both structural relaxation energy and spin-polarization energy. Shown in Table II, the magnitude of the structural distortion increases with supercell size, accompanied with greater relaxation energy, indicating stronger localization as finite size constraints are eased. The distortion parameter, the tetragonal distortion $c_{t} / a_{t}$ (see Fig. 4) for the $D_{2 d}$ structures and the axial disproportion ratio $c_{2} / c_{1}$ for the $C_{2 v}$ structure depart farther from symmetry as the size of the supercell increases, confirming greater localization as finite size effects are reduced.

The spin-polarization energy $\Delta E(\mathrm{sp})$ increases with supercell size also, shown in Table III. Greater spin-polarization energy indicates stronger localization in the unpaired spinorbital in the defect. These DFT results indicate the defect state in the infinitely dilute limit is indeed localized, leading to a distorted structure. The $\mathrm{rD}_{2 d}$ distortion in $\mathrm{Ga}_{A s}(1-)$ is already unmistakeable in the $3 \times 3 \times 3$ (216-atom) supercell.

TABLE III. Spin-polarization energy of $\mathrm{Ga}_{A s}(q)$, in $\mathrm{meV}$, as function of supercell. The energy change is with respect to a fully relaxed spinless ground state of the same symmetry.

\begin{tabular}{cccr}
\hline \hline Supercell & $\mathrm{r} D_{2 d}(1-)$ & $\mathrm{p} D_{2 d}(0)$ & $T_{d}(1+)$ \\
\hline & & $\mathrm{LDA}$ & \\
$3 \times 3 \times 3$ & -20 & -16 & +132 \\
$4 \times 4 \times 4$ & -21 & -14 & +32 \\
$5 \times 5 \times 5$ & -24 & -27 & -15 \\
& & $\mathrm{PBE}$ & \\
$3 \times 3 \times 3$ & -33 & -44 & +30 \\
$4 \times 4 \times 4$ & -38 & -53 & -58 \\
$5 \times 5 \times 5$ & -41 & -72 & -95 \\
\hline \hline
\end{tabular}

The removal of a second electron to create $\mathrm{Ga}_{A s}(0)$ accentuates the distortions seen in $\mathrm{Ga}_{A s}(1-)$. As presented in Table IV, the margin favoring the $C_{2 v}$ structure over the $T_{d}$ increases to $31 \mathrm{meV}$ for the $3 \times 3 \times 3$ supercell and $71 \mathrm{meV}$ for the $5 \times 5 \times 5$ supercell in the LDA, 64 increasing to $92 \mathrm{meV}$ in the PBE. The $\mathrm{r} D_{2 d}$ structure is even more stable, 51 through $84 \mathrm{meV}$ for LDA, 58 through $96 \mathrm{meV}$ for PBE. A proper nondegenerate spinless $C_{3 v}$ distortion also is lower than $T_{d}$, but is not competitive with these (these uncompetitive $C_{3 v}$ distorted structures are ignored henceforward). These energies, approaching $0.1 \mathrm{eV}$, unambiguously indicate a symmetry lowered ground state for $\mathrm{Ga}_{A s}(0)$. In $\mathrm{Ga}_{A s}(0)$, these distortions have no partially filled orbitals, and have no spin.

A spinless $\mathrm{p} D_{2 d}(0)$ structure is an improper state, but with two electrons in a degenerate doubleton of orbitals, a triplet spin state with one electron in each orbital results in a proper, nondegenerate total ground state. The LDA prefers $\mathrm{r} D_{2 d}(0)$, while PBE predicts this triplet-spin $\mathrm{p} D_{2 d}(0)$ to be lower. The differences, $30 \mathrm{meV}$ to one side or the other, are computationally significant, i.e., well-resolved numerically. In general, when DFT results hinge upon magnetism and spin polarization, PBE is more accurate than LDA. The cell dependence (in Table II) and the spin-polarization energy (Table III) confirm that this high-spin $\mathrm{p} D_{2 d}$ structure is a localized deep state. The overall preference between the two tetragonal distortions observed in these calculations is less than the physical accuracy observed with DFT in Si and GaAs, $0.1-0.2 \mathrm{eV}[5,15]$, so this difference is not necessarily

TABLE IV. Computed formation energy of $\mathrm{Ga}_{A s}(0)$, in $\mathrm{eV}$, as function of supercell, in As-rich (bulk $A 7$ structure) limit.

\begin{tabular}{lcccc}
\hline \hline Supercell & $T_{d}$ & $\mathrm{r} D_{2 d}$ & $\mathrm{p} D_{2 d}(\mathrm{sp})$ & $C_{2 v}$ \\
\hline & & \multicolumn{4}{c}{$\mathrm{LDA}$} \\
$2 \times 2 \times 2$ & 3.160 & 3.142 & 3.127 & 3.144 \\
$3 \times 3 \times 3$ & 3.186 & 3.135 & 3.141 & 3.155 \\
$4 \times 4 \times 4$ & 3.239 & 3.170 & 3.196 & 3.188 \\
$5 \times 5 \times 5$ & 3.261 & 3.177 & 3.208 & 3.190 \\
& & & $\mathrm{PBE}$ & \\
$2 \times 2 \times 2$ & 3.153 & 3.133 & 3.135 & 3.132 \\
$3 \times 3 \times 3$ & 3.196 & 3.138 & 3.120 & 3.152 \\
$4 \times 4 \times 4$ & 3.247 & 3.167 & 3.164 & 3.175 \\
$5 \times 5 \times 5$ & 3.271 & 3.175 & 3.170 & 3.179 \\
\hline \hline
\end{tabular}


physically meaningful, and the preference cannot be predicted with great confidence.

The trend of formation energies of $\mathrm{Ga}_{A s}$ as a function of supercell size is interesting. In every supercell sequence, independent of functional, $T_{d}$ or $\mathrm{r}_{2 d}$ or $\mathrm{p} D_{2 d}$ or $C_{2 v}$, presented in Table IV. The formation energy increases as supercell size increases. This observation is consequential for approaches that attempt to numerically extrapolate to an infinite bulk limit from results in a sequence of supercells [35-37]. The analytic expressions in these extrapolations are inspired by the Makov-Payne analysis [38] of electrostatic errors in finite supercells, and use that foundation to justify their form. In the $\mathrm{Ga}_{A s}(0)$ defects, there is no charge, and the charge multipole moments are minimal or zero, so the electrostatic corrections are minimal. Elastic relaxation effects would also lead to decreasing formation energy with increasing supercell size. This contrary trend must be due to spurious electronic interactions between defects, not anticipated in a Makov-Payne analysis. The exceptions to the trend, the $2 \times 2 \times 2$ (64-atom) supercell results for $\mathrm{r} D_{2 d}$ (LDA) and $\mathrm{p} D_{2 d}$ (PBE) neatly illustrate the extreme limit of this problem: The spurious interaction between periodic images of the defects has become so strong that the state occupations are corrupted, creating a mixed state inconsistent with a single asymptotic defect state.

The $\mathrm{Ga}_{A s}(+1)$ charge state does not allow a proper ground state in either $D_{2 d}$ distortion, the electron count mandates an occupation pattern with one electron or one hole in a degenerate doubleton, triggering a JT instability. Nonetheless, these symmetry-lowering distortions are lower than the spinless $T_{d}$. The highest occupied orbital in the $C_{2 v}$ distortion is nondegenerate; therefore, when occupied by one electron, constitutes a proper total state, and is also favored.

Alternatively, the three holes in $\mathrm{Ga}_{A s}(1+)$ can be distributed to each of the three degenerate $t_{2}$ orbitals in the $T_{d}$ structure. With one electron in each orbital, high-spin coupled, the total state is nondegenerate and not JT unstable. In the smaller supercells, this high-spin $T_{d}$ state has a higher computed energy than the spinless $T_{d}$, by $142 \mathrm{meV}$ in LDA and $28 \mathrm{meV}$ in $\mathrm{PBE}$ for the $3 \times 3 \times 3$ supercell, but becomes favored over spinless $T_{d}$ in the $4 \times 4 \times 4$ supercell with $\mathrm{PBE}$, and in both LDA and PBE in the largest $5 \times 5 \times 5$ supercell, by 15 and $95 \mathrm{meV}$, respectively. This large supercell dependence with spin polarization indicates finite-size errors are dominated by electronic interactions between defects rather than the Coulombic interaction between electrostatic multipole moments. The crowding of the orbitals confined in the smaller cells makes the exchange coupling unfavorable, while in the larger supercells all the orbitals localize within the volume of the supercell and thereby high-spin couple without interference.

For the LDA, this high-spin $T_{d}$ structure gains upon, but does not become more stable than, the $C_{2 v}$ structure in even the largest supercell considered here. The PBE clearly favors the $T_{d}$ structure in the asymptotic bulk limit. As before, if the nature of the defect is dependent upon spin, PBE is usually more accurate; the high-spin $T_{d}$ is likely the ground state structure for $\mathrm{Ga}_{A s}(1+)$. It is possible that this would become favored in LDA in even larger supercells, the margin between it and $C_{2 v}$ is still narrowing considerably with the 1000-atom supercell.
The level occupation patterns for $\mathrm{Ga}_{A s}(2+)$ allow a proper state for a spin-zero $\mathrm{p} D_{2 d}$, a spin-triplet $\mathrm{r} D_{2 d}$, and a spin-zero $C_{2 v}$ structure. None of these result in significant distortion from $T_{d}$. For the smallest 216-atom cells, the $C_{2 v}$ and $\mathrm{p} D_{2 d}$ distortions collapsed to a $\sim T_{d}$ structure. The triplet-spin $\mathrm{r} D_{2 d}(2+)$ structure shows minimal distortion from the spin$\frac{3}{2} T_{d}(1+)$ structure. It is only with the 1000 -atom supercell that the $\mathrm{p} D_{2 d}$ opens a nonnegligible margin, becoming $15 \mathrm{meV}$ more stable than $T_{d}$. The $\mathrm{p} D_{2 d}$ distortion becomes smaller with increased supercell size, as presented in Table II, suggesting it might be trending toward a symmetric structure indicative of a true shallow state or an unphysical band edge state.

The results for $\mathrm{Ga}_{A s}(2+)$ suggest it is possibly a shallow state, and, at least, that a structural analysis by itself is inconclusive in discriminating its character. For each of the $(2+)$ structures, it becomes problematic to cleanly distinguish defect eigenstates from the VBE, and this near degeneracy is reflected in the lack of large distortion energies. In the other charge states, the distinction between the defect eigenstates and the VBE is clearer. While the DFT eigenvalues are not useful for distinguishing total energies (and charge transition defect level energies), they are crucial in distinguishing the character of the states associate with those orbitals.

\section{Shallow acceptors}

Before examining the DFT state levels in the Ga antisite, it is instructive to consider the computational behavior of known shallow acceptors in GaAs. Boron is isovalent with gallium, and its substitution for arsenic in the lattice also leads to two missing electrons in the bonds. Carbon is a common $p$-type dopant in GaAs, a shallow single acceptor. How do these substitutions on the As lattice site, known shallow acceptors, behave in supercell calculations?

Concerted attempts to find symmetry-lowered ground states were unsuccessful for these shallow acceptors, to within the practical resolution of the DFT calculations $(\sim 1-2 \mathrm{meV})$. A $T_{d}$ configuration was the only stable structure found for every nominal charge state. The change in nearest neighbor bond lengths with change in charge state is very small, as shown in Table V. Only the PBE results are presented, the LDA leads to the same analysis. In contrast, the bond distances for $\mathrm{Ga}_{A s}$ presented in Table I exhibited 1\%-2\% increases in bond distances with each change in charge state, rather than the $0.1 \%-0.2 \%$ changes seen for $\mathrm{B}_{A s}$ and $\mathrm{C}_{A s}$.

The contrast between $\mathrm{Ga}_{A s}$ and these shallow acceptors is even more stark using the magnitude of spin-polarization energy in the single hole state. For the shallow acceptors, the spin-polarization energy $\Delta E_{\mathrm{sp}}$ is $3 \mathrm{meV}$ at most. Spin stabilization is largest in the smallest $3 \times 3 \times 3$ cells and then decreases with increasing supercell size, to $1 \mathrm{meV}$ or less. This is consistent with a strongly delocalized hole state, becoming more delocalized with increasing supercell size. In contrast, the spin-eligible $\mathrm{Ga}_{A s}$ defects had spin stabilization energies worth 10's of meV, increasing with increasing supercell size, indicating greater localization of the defect state.

It is plausible that the large strain induced by first row atoms overwhelms potential distortions from symmetry, that $\mathrm{Ga}$ is different because of its size, being able to accommodate local distortions without competition from lattice strain. The 
TABLE V. Computed $R_{X-G a}$ bond distances (in $\AA$ ) for shallow substitutional $X_{A s}$ defects $(X=\mathrm{B}, \mathrm{C}, \mathrm{Ge})$, using the PBE functional, with supercell size. Spin polarized is denoted by "sp."

\begin{tabular}{lccc}
\hline \hline & \multicolumn{3}{c}{ Supercell } \\
\cline { 2 - 4 } Defect & $3 \times 3 \times 3$ & $4 \times 4 \times 4$ & $5 \times 5 \times 5$ \\
\hline $\mathrm{B}_{A s}$ & & & \\
$(2-)$ & 2.096 & 2.097 & 2.096 \\
$(1-)$ & 2.104 & 2.101 & 2.101 \\
$\mathrm{sp}(1-)$ & 2.104 & 2.101 & 2.101 \\
$(0)$ & 2.109 & 2.105 & 2.103 \\
$\Delta E_{\mathrm{sp}}(\mathrm{meV})$ & -3.2 & -2.0 & -1.6 \\
$\mathrm{C}_{A s}$ & & & \\
$(1-)$ & 2.059 & 2.058 & 2.058 \\
$(0)$ & 2.059 & 2.058 & 2.058 \\
$\mathrm{sp}(0)$ & 2.059 & 2.058 & 2.058 \\
$\Delta E_{\mathrm{sp}}(\mathrm{meV})$ & -1.8 & -0.8 & -0.4 \\
$\mathrm{Ge}_{A s}$ & & & \\
$(1-)$ & 2.427 & 2.428 & 2.428 \\
$(0)$ & 2.434 & 2.432 & 2.432 \\
$\mathrm{sp}(0)$ & 2.435 & 2.432 & 2.432 \\
$\Delta E_{\mathrm{sp}}(\mathrm{meV})$ & -2.6 & -1.2 & -0.7 \\
\hline \hline
\end{tabular}

results for $\mathrm{Ge}_{A s}$, also presented in Table $\mathrm{V}$, dispel this notion. Germanium is to gallium what carbon is to boron, in the same row of the periodic table as Ga and roughly the same size, and $\mathrm{Ge}$ is known to be a shallow acceptor $(<40 \mathrm{meV}$ above the VBE) [39]. The $\mathrm{Ge}_{A s}$ shows no hint of structural distortion, bond lengths do not change with change in nominal charge state, and the spin-polarization energy is very small and decreases with supercell size. The $\mathrm{Ga}_{A s}$ is distinct from $\mathrm{Ge}_{A s}$ in a way that $\mathrm{B}_{A s}$ is not distinct from $\mathrm{C}_{A s}$. The $\mathrm{Ge}_{A s}$ has qualitative features in common with the other known shallow defects; $\mathrm{Ga}_{A s}$ is qualitatively different.

The nominal charge states for these shallow defects do not represent local defects, instead, they represent fully charged defects with holes in the VB. Examination of the DFT orbital eigenstates in each of the supercell calculations lends support to this interpretation. While the DFT eigenvalues (and their relationship to VBE) do not have any meaningful relationship to the total energies and defect level positions of the associated defect, they can be used to discriminate the character of a defect state and identify states that are potentially suspect. Here, also, the $\mathrm{Ga}_{A s}$ is qualitatively different than the shallow acceptors.

\section{Orbital eigenstate level analysis}

An eigenstate analysis is complicated by use of finite supercells. First, defects, instead of expressing discrete levels, interact with their periodic images, causing artificial banding in the defect orbital eigenstate. Second, because of the finite size of the cell, all the states within the cell, including the nominal crystal states, are perturbed from the perfect crystalline band structure. Third, to perform sufficiently accurate integration within the Brillouin zone (BZ), a nontrivial sampling of $k$ points is required: a $2^{3}$ grid in each of the larger cells $\left(3^{2}\right.$ in the smaller 64 -atom $2 \times 2 \times 2$ supercell). It proves computationally convenient to offset this $k$-sample grid from the $\Gamma$ point to a body-center point in the grid, the resulting

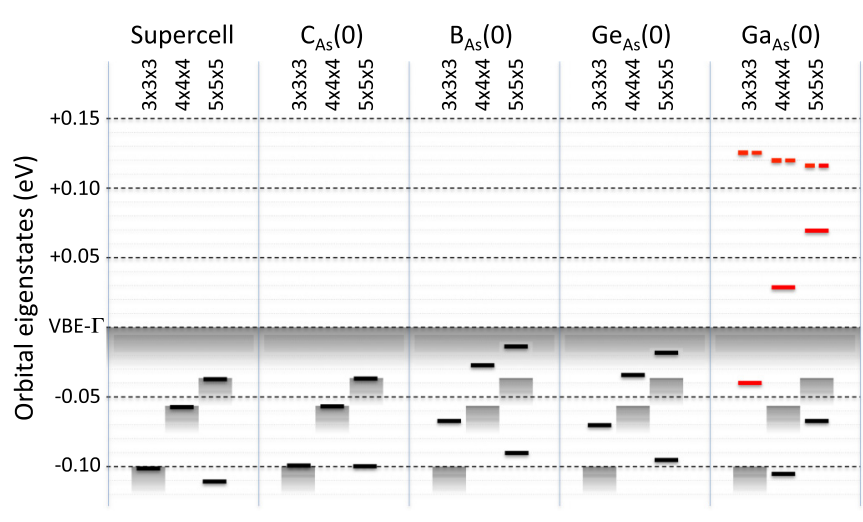

FIG. 5. Orbital eigenvalues of states near the VBE for the perfect supercell and a sequence of neutral $T_{d}$ substitutional defects, computed with PBE. While the shallow acceptors perturb the highest VB-like states above VBE at the $k$-sampling point for their respective cells $\left(\mathrm{C}_{A s}\right.$ negligibly so), these do not rise above the global VBE at $\Gamma$. The highest levels in $\mathrm{Ga}_{A s}$ are defect states (in red), split into a doubleton over a singleton by the off- $\Gamma k$ point, are converging with increasing supercell size to a degenerate tripleton above $\operatorname{VBE}(\Gamma)$.

sampling in high-symmetry $T_{d}$ or $D_{2 d}$ consists of just a single $k$ point in the irreducible $\mathrm{BZ}$, reduced from eight $k$ points in the full zone. This also frequently proves physically convenient, as a band gap problem can result in sampling errors at $\Gamma$, where defect states might disappear from the gap into the bulk band structure (this is not an issue for $\mathrm{Ga}_{A s}$ and the shallow defects considered here). The occupation method ensures that the correct eigenstates are occupied, defect band states that asymptotically map onto the infinitely dilute limit of isolated defect states.

Figure 5 illustrates the eigenvalue diagrams at the sampled $k$ point for acceptor defects in their relaxed $T_{d}$ structures: shallow acceptors $\mathrm{C}_{A s}(0), \mathrm{B}_{A s}(0)$, and $\mathrm{Ge}_{A s}(0)$, and the neutral gallium antisite. To establish a common reference across the different defects and different supercells, the eigenstates are referenced to the global VBE, at $\Gamma$. The $\operatorname{VBE}(k)$ is not a consistent marker, the $k$ sample in each supercell is at a different point in the BZ. The VBE state at each defect calculation is perturbed by the acceptor state being embedded in the bonding network. The $\mathrm{CBE}(k)$ in each neutral defect calculation proves to be very stable, taking the same eigenvalue as in the perfect crystal supercell, to within 2-3 meV for every defect calculation examined here. This $\operatorname{CBE}(k)$ in each calculation is used as a marker to locate $\operatorname{VBE}(\Gamma)$.

Seen in Fig. 5, $\mathrm{C}_{A s}$ adopts a state structure almost identical to the perfect crystal supercell, which indicates a hole in a band edge state rather than a localized defect state. Its shallow acceptor state is buried below the VBE, indistinguishable from bulk band states, even in the 1000-atom supercell.

The $\mathrm{B}_{A s}(0)$ also mimics the perfect crystal supercell levels. While the more perturbed states at the VBE are shifted higher than $\operatorname{VBE}(k)$, these are nonetheless VBE-derived states, there are no distinct new states to be associated with a defect. The shallow acceptor state is still inextricably embedded in the valence bands in these small supercells.

The shifts in the VB eigenstates for $\mathrm{Ge}_{A s}(0)$ are similar to $\mathrm{B}_{A s}$, it has no new eigenstates that could be associated with 


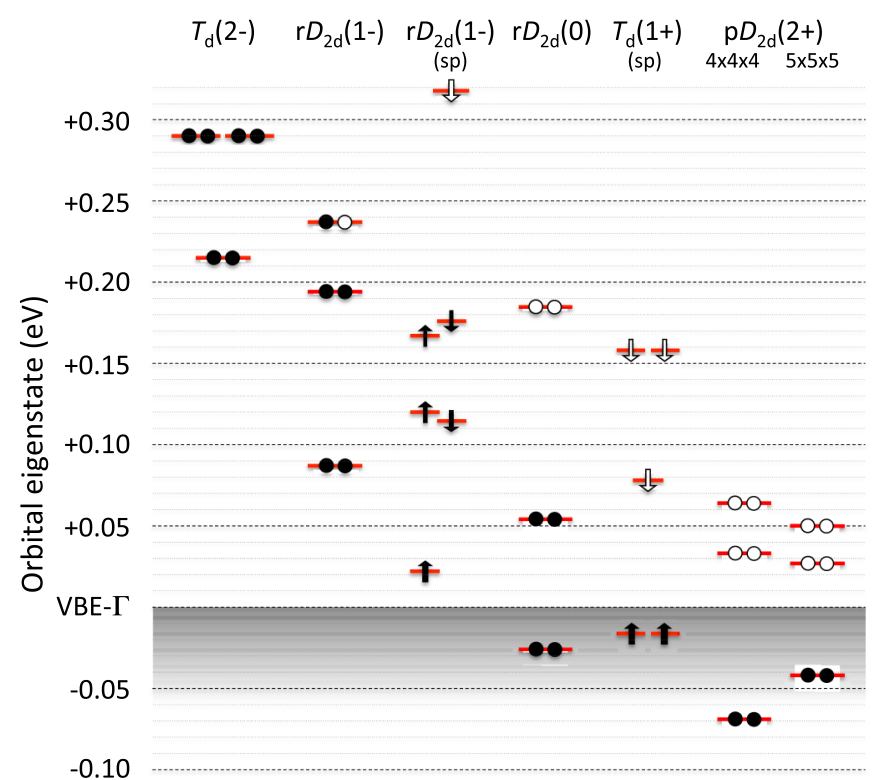

$-0.15$

FIG. 6. Orbital eigenstates and level occupations for $\mathrm{Ga}_{A s}(q)$ defects, with the PBE functional in the 512-atom supercell. The eigenvalues are referenced to the VBE at the $\Gamma$ point (see text). The hole states all are above the VBE for each charge state, consistent with a deep local defect, although the $(2+)$ is somewhat marginal, with the larger supercell hole states converging toward the VBE.

a defect state. In each of these cases, these small supercells fail to clearly distinguish the acceptor states from the VBE, "small" extending past 1000-atom supercells.

The $\mathrm{Ga}_{A s}(0)$ is qualitatively different. Perfect crystal VBE eigenstates are present, but shifted deeper relative the supercell $\operatorname{VBE}(k)$. New states have appeared, well within the global band gap, clearly distinct from these crystal-derived states. A degenerate doubleton appears $0.12 \mathrm{eV}$ above the $\mathrm{VBE}$ at $\Gamma$. A third defect orbital appears above $\operatorname{VBE}(k)$, and then rapidly approaches the degenerate doubleton as the supercell size increases. These constitute the expected tripleton of degenerate $p$-type orbitals, that the off- $\Gamma k$ point has split. This $k$ splitting is a crude measure of the magnitude of the artificial defect banding engendered by the finite supercell. A splitting of $0.2 \mathrm{eV}$ in the 216-atom cell indicates rather significant defect band dispersion, reduced to $0.05 \mathrm{eV}$ in the 1000 -atom cell. The appearance of these new levels in the gap, and trend with increasing cell size, is an unambiguous indicator that the $\mathrm{Ga}_{A s}$ is a deep defect.

Figure 6 presents similar eigenstate diagrams for a sequence of charge states in $\mathrm{Ga}_{A s}$, within the $4 \times 4 \times 4$ 512-atom supercell. As before, the $\operatorname{CBE}(k)$ is the marker used in each supercell defect calculation to calibrate the global $\operatorname{VBE}(\Gamma)$ position. The $\mathrm{Ga}_{A s}(2-)$ remains $T_{d}$, and adopts a level pattern similar to the $T_{d} \mathrm{Ga}_{A s}(0)$ shown in Fig. 5. The eigenstates are shifted higher than in the (0) by almost $0.2 \mathrm{eV}$, reflecting the increased occupation in the localized orbital of the charged defect, with an eigenstate that is $0.28 \mathrm{eV}$ above $\operatorname{VBE}(\Gamma)$.
Removing an electron to obtain the $\mathrm{Ga}_{A s}(1-)$ causes a r $D_{2 d}$ distortion. The highest partially occupied state is $0.24 \mathrm{eV}$ above the $\operatorname{VBE}(\Gamma)$ for the spinless calculation, reduced from $0.28 \mathrm{eV}$ in the (2-) defect. Introducing spin polarization causes a significant split between the majority- and minority-spin levels. The highest occupied state is $0.18 \mathrm{eV}$ above the band edge, while the newly empty state has risen to $0.31 \mathrm{eV}$ above the VBE. The magnitude of spin splitting is further evidence of a localized state.

The eigenstates shift further toward the VBE in the $\mathrm{r} D_{2 d}(0)$ structure. The emptied eigenstate is now $0.19 \mathrm{eV}$ above the VBE, and one orbital of the $k$-split degenerate doubleton has dipped below $\operatorname{VBE}(\Gamma)$. The overall shift in the defect states is $\sim 50 \mathrm{meV}$, again indicating localization in this state.

$\mathrm{The} \mathrm{Ga}_{A s}(1+)$ ground state, the spin- $\frac{3}{2} T_{d}$ structure, is rather distinctive. All occupied majority-spin defect states are below the $\operatorname{VBE}(\Gamma)$, one $k$-split member of the degenerate tripleton significantly below $\operatorname{VBE}(k)$. The empty, minority-spin orbital eigenstates are well above the VBE. These orbitals levels for $\mathrm{Ga}_{A s}(1+)$ indicate a localized defect state, but beg the question whether yet another electron could be removed; all occupied orbitals are now below the global VBE.

The $\mathrm{p} D_{2 d}$ distortion causes more eigenstate shifts in $\mathrm{Ga}_{A s}(2+)$, leaving all empty orbitals above the VBE and all occupied orbitals below it. The margins are rather small, the empty state is only $33 \mathrm{meV}$ above $\operatorname{VBE}(\Gamma)$, and this state trends toward the VBE with increasing supercell size. With such small, shrinking margins, characterization of $\mathrm{Ga}_{A s}(2+)$ as a localized defect cannot be made with the same confidence.

This eigenstate analysis identifies the $\mathrm{Ga}_{A s}$ as a deep defect, with charge states ranging from (2-) through $(2+)$, with a possibility that the $(2+)$ is a shallow state. This assessment is consistent with the total energy calculations and structural analyses presented earlier. This eigenstate analysis, within the conceptual framework of the occupation level pattern, reliably discriminates the deep defect nature of $\mathrm{Ga}_{A s}$.

\section{E. Defect levels}

The DFT eigenstates cannot be used in lieu of total energy calculations in computing the defect level transitions. In Table VI the computed defect energy levels for $\mathrm{Ga}_{A s}$ in $\mathrm{GaAs}$ are computed from total energy differences, using the fully relaxed, reduced-symmetry ground state structure for each charge state and supercell, including spin polarization. The results from each supercell are extrapolated to an infinitely dilute limit using the LMCC and finite cell polarization corrections [15,32,33]. The progression across supercells shows that the computed defect levels are well converged to the bulk asymptotic limit with 512-atom supercells. The computed levels for the 512-atom and 1000-atom supercells mostly match to within the quoted $0.01 \mathrm{eV}$ resolution.

The $(2-/ 1-)$ transition is midgap, the $(1-/ 0)$ and $(0 / 1+)$ forming a sequence of levels going downwards spaced $\sim 0.2 \mathrm{eV}$ apart. These predicted defect levels are far from the band edges, more than the $0.2 \mathrm{eV}$ accuracy observed for levels in GaAs [5,16], and are predicted to be deep defect states with high confidence. The computed $(1+/ 2+)$ level, in both LDA and PBE, is within $\sim 0.2 \mathrm{eV}$ of the VBE, so this prediction is less certain. 
TABLE VI. Computed defect levels of $\mathrm{Ga}_{A s}$, in $\mathrm{eV}$, with respect to the valence band edge.

\begin{tabular}{lcccc}
\hline \hline & \multicolumn{5}{c}{ Charge transition } \\
\cline { 2 - 5 } Supercell & $(2-/ 1-)$ & $(1-/ 0)$ & $(0 / 1+)$ & $(1+/ 2+)$ \\
\hline \multicolumn{5}{c}{ LDA } \\
$3 \times 3 \times 3^{\mathrm{a}}$ & 0.77 & 0.57 & 0.40 & 0.26 \\
$3 \times 3 \times 3$ & 0.80 & 0.59 & 0.37 & $\mathrm{~b}$ \\
$4 \times 4 \times 4$ & 0.78 & 0.58 & 0.35 & 0.24 \\
$5 \times 5 \times 5$ & 0.78 & 0.57 & 0.34 & 0.25 \\
& & & PBE & \\
$3 \times 3 \times 3^{\mathrm{a}}$ & 0.78 & 0.60 & 0.44 & 0.29 \\
$3 \times 3 \times 3$ & 0.83 & 0.63 & 0.39 & $\mathrm{~b}$ \\
$4 \times 4 \times 4$ & 0.81 & 0.60 & 0.39 & 0.22 \\
$5 \times 5 \times 5$ & 0.81 & 0.59 & 0.41 & 0.22 \\
\hline \hline
\end{tabular}

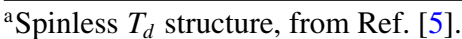

${ }^{\mathrm{b}}$ Not stable as a local defect.

Interestingly, the original $\mathrm{Ga}_{A s}$ defect levels computed using $T_{d}$ structures [5] are very similar, the structural distortion and spin-polarization energies are rather modest. The important consequence of this more refined analysis is the quantitative confidence that can be made concerning the qualitative characterization of $\mathrm{Ga}_{A s}$.

Defect results for GaAs are typically presented in the arsenic-rich limit, to match the growth conditions prevalent in most manufacture of GaAs. In Table VII the formation energies of simple intrinsic defects in GaAs are instead presented in the Ga-rich limit, with the Fermi level set to the VBE, to be consistent with the growth conditions in which the 78/203 double acceptor is observed. The conversion from the As-rich to the Ga-rich limit is straightforward within the prescription of Zhang and Northrup [40], requiring knowledge of the GaAs heat of formation, here 0.740 and $0.787 \mathrm{eV}$ with the LDA and PBE functionals, respectively. Results for the 512-atom cells with PBE are presented, this cell size is converged to the asymptotic limit. The LDA results are similar.

The formation energies of $\mathrm{Ga}_{A s}$ are presented using the spin-resolved results, the other defects use spin-restricted results obtained previously [5,41]. Also presented in Table VII

TABLE VII. Formation energies of intrinsic defects in GaAs, in $\mathrm{eV}$, using the PBE functional in $4 \times 4 \times 4$ (512-site) cubic supercells, with the Fermi level set to the valence band edge, in the Ga-rich (bulk $A 11$ structure) limit.

\begin{tabular}{lcccccccc}
\hline \hline & \multicolumn{7}{c}{ Charge state } \\
\cline { 2 - 8 } Defect & $(3+)$ & $(2+)$ & $(1+)$ & $(0)$ & $(1-)$ & $(2-)$ & $(3-)$ & $(4-)$ \\
\hline $\mathrm{Ga}_{A s}$ & - & 0.92 & 1.14 & 1.52 & 2.11 & 2.93 & - & - \\
$\mathrm{sh}_{-G a} \mathrm{Ga}_{A s}$ & - & - & - & 2.64 & 2.72 & 2.93 & - & - \\
$\mathrm{As}_{G a}$ & - & 1.69 & 2.19 & 2.92 & - & - & - & - \\
$v_{G a}$ & 2.17 & 2.74 & 2.88 & 3.53 & 4.02 & 4.64 & 5.42 & - \\
$v_{A s}$ & 1.04 & 1.53 & 1.56 & 2.62 & 3.28 & 4.71 & 5.61 & - \\
$v v$ & - & 3.09 & 3.30 & 3.62 & 4.70 & 5.29 & 6.87 & 8.38 \\
$\mathrm{As}_{i}$ & 3.08 & 3.43 & 3.18 & 4.27 & 5.14 & - & - & - \\
$\mathrm{Ga}_{i}$ & 1.12 & 1.24 & 1.35 & 3.36 & 4.48 & - & - & - \\
$a a$ & - & - & - & 2.44 & - & - & - & - \\
\hline \hline
\end{tabular}

are the formation energies of a hypothetical "sh-Ga $\mathrm{Ga}_{A s}$," that sets aside the conclusions from the above analysis and instead posits that the $\mathrm{Ga}$ antisite is the $78 / 203$ shallow acceptor. For sh-Ga $\mathrm{Ga}_{A s}$, the $(0)$ and $(1-)$ charge states are deemed embedded in the VBE and the directly computed energies are meaningless. The formation energies for $\mathrm{sh}-\mathrm{Ga}_{A s}$ are computed via the (2-) charge state, a valid defect state, then sequentially using the experimental shallow defect level energies, 78 and $203 \mathrm{meV}$, to obtain the formation energies of the (1-) and (0) charge states.

\section{F. The $\mathbf{G a}_{A s}$ and the 78/203 shallow double acceptor}

The LDA and PBE calculations for $\mathrm{Ga}_{A s}$ clearly exhibit significant distortions from the $T_{d}$ structure, consistent with the LCAO-MO analysis of a localized state. However, the accuracy of semilocal functionals is often impugned due to their shared failure to predict the band gap. The exchange mixing parameter of hybrid functionals can be empirically tuned to have its eigenstate gap match the experimental band gap, and it might be hypothesized that a more accurate treatment would obtain a different result. For $\mathrm{Ga}_{A s}$, the LDA and PBE functionals already localize the defect and find states to be in the gap, and where these find localized states, they can be used for quantitative predictions $[5,15,16]$. Hybrid calculations for $T_{d} \mathrm{Ga}_{A s}$ [12] find qualitatively similar results to those obtained with PBE and LDA.

Additional evidence that the band gap problem is inconsequential for $\mathrm{Ga}_{A s}$ is that the eigenstates for different $\mathrm{Ga}_{A s}$ charge states are fully contained with the the DFT band gap ( $0.45 \mathrm{eV}$ for PBE GaAs), $0.28 \mathrm{eV}$ above the VBE for the $\mathrm{GaAs}_{A s}(2-)$, while the computed transition energies range up to $0.8 \mathrm{eV}$ above the VBE. The defect eigenstates energies are disconnected from the energy levels computed from total energy differences, the computed defect levels in GaAs. The total energy scale for defect levels is greatly expanded from the orbital eigenstates scale, a general result in DFT studies of defects. This corroborates earlier observations that all defect levels in GaAs (and $\mathrm{Si}$ ) are mostly insensitive to variation in the DFT eigenvalue band gap [22].

For the 78/203 shallow double acceptor, Elliott et al. [8] had determined that any distortion was negligible, that the linewidth in their measurements precluded distortion energies larger than $0.5 \mathrm{meV}$. This lack of distortion is seen in the known shallow defects $\mathrm{B}_{A s}, \mathrm{C}_{A s}$, and $\mathrm{Ge}_{A s}$. The symmetry of the $78 / 203$ is tetrahedral, consistent with the $\mathrm{B}_{A s}$ results obtained with DFT above, and inconsistent with the large distortions for $\mathrm{Ga}_{A s}$, approaching $0.1 \mathrm{eV}$, obtained with DFT.

Computed defect formation energies add more evidence $\mathrm{Ga}_{A s}$ cannot be the shallow double acceptor. The 78/203 defect only appears in Ga-rich GaAs, seemingly implicating a Ga-rich defect: $\mathrm{Ga}_{A s}, v_{A s}$, or $\mathrm{Ga}_{i}$. The formation energy of the DFT-predicted deep double donor $\mathrm{Ga}_{A s}$ is the lowest energy defect, but a shallow $\mathrm{Ga}_{A s}$ model for the 78/203 defect is clearly disfavored in thermally grown Ga-rich material. Assignment of the 78/203 shallow double acceptor to $\mathrm{Ga}_{A s}$ depends upon the assumption that $\mathrm{Ga}_{A s}$ is a low-energy defect. A sh- $\mathrm{Ga}_{A s}$ is a high-energy defect in Ga-rich GaAs, even the As antisite is lower in $p$-type GaAs. The most common intrinsic defects in $p$-type Ga-rich GaAs, instead of sh-Ga ${ }_{A s}$, would 
be $v_{A s}(3+)$ or $\mathrm{Ga}_{i}(3+) . \mathrm{Ga}_{A s}$ is competitive with these only identified as the deep double donor predicted with DFT. With formation energies near $1 \mathrm{eV}$, none of these would be populous. Because they are deep donors, they would compensate any shallow acceptors, and tend toward semi-insulating native material, again in agreement with observations of the most recent experiments [11]. Treating the $3 d$ electrons as valence electrons reduces these formation energies somewhat $[5,41]$, but does not materially affect this conclusion.

\section{SUMMARY AND CONCLUSIONS}

A careful analysis using DFT supercell calculations clearly excludes the $\mathrm{Ga}_{A s}$ as a candidate for the 78/203 shallow double acceptor in Ga-rich GaAs, contrary to a recent bounds analysis interpretation [13]. The computed distortions, level analysis, defect levels, and formation energies show $\mathrm{Ga}_{A s}$ to be a deep defect. The $\mathrm{B}_{A s}$, conversely, is shown to be embedded in the VBE, a shallow effective-mass-like defect, consistent with the $78 / 203$ shallow double acceptor, confirming a recent experimental identification.

This assessment is enabled by a level occupation analysis, mapping of the defect states onto a conceptual LCAO-MO framework, which identified the viable structures and spin states to explore for each nominal charge state. The $\mathrm{Ga}_{A s}$ is not the 78/203 defect, nor is it shallow. An analysis of the structural changes and distortions indicated localized charge states for $\mathrm{Ga}_{A s}$, ranging from (2-), acceptor states, through $(2+)$, donor states, predicting two, deep acceptor levels and also two donor levels, one of which might be shallow. Using the DFT functional as a laboratory, evaluation of spin-polarization energies discriminated localized orbitals from delocalized band states, and gave assessments consistent with the structural analyses. The holes in the bonds to the antisite atom trigger a sequence of modest but meaningful distortions.

The analysis of the orbital eigenstate energies was additional evidence of the character of $\mathrm{Ga}_{A s}$, further buttressed by comparisons to known shallow acceptors. The orbital eigenstates of $\mathrm{Ga}_{A s}$ were within the DFT band gap. An interesting consequence of this analysis is that the total energy calculations produced levels in the gap that far exceeded the positions of the associated orbital eigenstates in the DFT eigenvalue gap, providing an example of how the orbital gap is not a meaningful measure nor constraint on defect level calculations.

\section{ACKNOWLEDGMENTS}

I am grateful to Art Edwards for many useful discussions regarding modeling defects, and for a critical reading of the manuscript. Sandia is a multiprogram laboratory managed and operated by Sandia Corporation, a wholly owned subsidiary of Lockheed Martin Company, for the United States Department of Energy's National Nuclear Security Administration under Contract DE-AC04-94AL85000.
[1] P. A. Schultz, J. Rad. Effects Res. Eng. 30, 257 (2012), for a copy contact the DTRA Defense Threat Reduction Information Analysis Center (DTRIAC), Attn: Documents Manager, 1680 Texas Street SE, Kirtland AFB, NM 87117-5669; Sandia National Laboratories Report No. SAND2012-6659 (2012).

[2] W. R. Wampler and S. M. Myers, J. Appl. Phys. 117, 045707 (2015).

[3] D. Pons and J. C. Bourgoin, J. Phys. C: Solid State Phys. 18, 3839 (1985).

[4] J. C. Bourgoin, H. J. Bardeleben, and D. Stiévenard, J. Appl. Phys. 64, R65 (1988).

[5] P. A. Schultz and O. A. von Lilienfeld, Modell. Simul. Mater. Sci. Eng. 17, 084007 (2009).

[6] J. Dabrowski and M. Scheffler, Phys. Rev. Lett. 60, 2183 (1988).

[7] D. J. Chadi and K. J. Chang, Phys. Rev. Lett. 60, 2187 (1988).

[8] K. R. Elliott, D. E. Holmes, R. T. Chen, and C. G. Kirkpatrick, Appl. Phys. Lett. 40, 898 (1982).

[9] P. W. Yu, W. C. Mitchell, M. G. Mier, S. S. Li, and L. Wang, Appl. Phys. Lett. 41, 532 (1982).

[10] K. R. Elliott, Appl. Phys. Lett. 42, 274 (1983).

[11] F.-M. Kiessling, M. Albrecht, K. Irmscher, R. Kraus-Rehberg, W. Ulrici, and P. Rudolph, J. Cryst. Growth 310, 1418 (2008); F. M. Kiessling, N. Albrecht, K. Irmscher, M. Roßberg, P. Rudolph, W. Ulrici, and R. Fornari, Phys. Status Solidi C 6, 2778 (2009).

[12] H. K. Komsa and A. Pasquarello, Physica B 407, 2833 (2012).
[13] A. F. Wright and N. A. Modine, Phys. Rev. B 91, 014110 (2015).

[14] L.-W. Wang, J. Appl. Phys. 105, 123712 (2009).

[15] P. A. Schultz, Phys. Rev. Lett. 96, 246401 (2006).

[16] P. A. Schultz, J. Phys.: Condens. Matter 27, 075801 (2015).

[17] K. R. Elliott, J. Appl. Phys. 55, 3856 (1984).

[18] G. Roos, A. Schöner, and G. Pensl, J. Appl. Phys. 69, 1454 (1991).

[19] R. Addinall, R. C. Newman, W. Götz, G. Roos, G. Pensl, and D. A. Hope, Mater. Sci. Forum 65, 175 (1991).

[20] R. Addinall and R. C. Newman, Semicond. Sci. Technol. 7, 1005 (1992).

[21] M. Suezawa and K. Sumino, J. Appl. Phys. 76, 932 (1994).

[22] P. A. Schultz and A. H. Edwards, Nucl. Instrum. Methods B 327, 2 (2014).

[23] G. D. Watkins, in Deep Centers in Semiconductors, edited by S. T. Pantelides (Gordon and Breach, New York, 1986), p. 177.

[24] H. Jahn and E. Teller, Proc. R. Soc. London Ser. A 161, 220 (1937).

[25] R. P. Messmer, B. McCarroll, and C. M. Singal, J. Vac. Sci. Technol. 9, 891 (1972).

[26] P. A. Schultz, SEQQUEST code (unpublished), see http://dft. sandia.gov/Quest/.

[27] J. P. Perdew and A. Zunger, Phys. Rev. B 23, 5048 (1981).

[28] J. P. Perdew, K. Burke, and M. Ernzerhof, Phys. Rev. Lett. 77, 3865 (1996).

[29] A. E. Mattsson, P. A. Schultz, T. R. Mattsson, K. Leung, and M. P. Desjarlais, Model. Simul. Mater. Sci. Eng. 13, R1 (2005). 
[30] O. A. von Lilienfeld and P. A. Schultz, Phys. Rev. B 77, 115202 (2008).

[31] S. G. Louie, S. Froyen, and M. L. Cohen, Phys. Rev. B 26, 1738 (1982).

[32] P. A. Schultz, Phys. Rev. B 60, 1551 (1999).

[33] P. A. Schultz, Phys. Rev. Lett. 84, 1942 (2000).

[34] I. Vurgaftman, J. R. Meyer, and L. R. Ram-Mohan, J. Appl. Phys. 89, 5815 (2001).

[35] C. W. M. Castleton and S. Mirbt, Phys. Rev. B 70, 195202 (2004).
[36] C. W. M. Castleton, A. Höglund, and S. Mirbt, Phys. Rev. B 73, 035215 (2006).

[37] N. D. M. Hine, K. Frensch, W. M. C. Foulkes, and M. W. Finnis, Phys. Rev. B 79, 024112 (2009).

[38] G. Makov and M. C. Payne, Phys. Rev. B 51, 4014 (1995).

[39] F. E. Rosztoczy, F. Ermanis, I. Hayashi, and B. Schwartz, J. Appl. Phys. 41, 264 (1970).

[40] S. B. Zhang and J. E. Northrup, Phys. Rev. Lett. 67, 2339 (1991).

[41] P. A. Schultz, Sandia National Laboratories Report No. SAND2012-2675 (April, 2012). 